\title{
Aproximación socio-epistemológica: fundamento para propuestas didácticas relativas al concepto de serie
}

\author{
José Leonardo Ángel Bautista \\ Óscar Javier Molina Jaime ${ }^{1}$ \\ Artículo recibido el 13 de junio de 2008 y aprobado el 27 de noviembre de 2008. \\ Socioepistemological approach: referent for \\ didactical proposals about the series concept
}

Resumen: En este escrito se reporta parte de la investigación que se desarrolló en el marco del trabajo de maestría (elaborado en 2007 como requisito parcial para obtener el titulo de Magíster en Docencia de las Matemáticas en la Universidad Pedagógica Nacional) elaborado por los autores y titulado "Influencia de las series en la evolución del concepto de función: una aproximación socio-epistemológica". Se expone un análisis fundamentado en la propuesta teórico-metodológica"aproximación socio-epistemológica" para la investigación en educación matemática. Específicamente, se ilustra cómo ciertos factores sociales en una época determinada (en este caso el siglo XVII) influyen en el desarrollo de los conceptos matemáticos de serie y función, y cómo un análisis de tales hechos contribuye al diseño de propuestas didácticas para el estudio de dichos conceptos en el aula.

Palabras clave: Aproximación socio-epistemológica, serie, función.
Abstract: In this document we report part of the research that was developed in the master's (work elaborated in 2007 like partial requirement to obtain the title of Master in Mathematical Teaching in Universidad Pedagógica Nacional.) project made by the authors and called "Influencia de las series en la evolución del concepto de función: una aproximación socio-epistemológica". In this report, we present an analysis based on the "socioepistemological approach", a methodological-theory proposal for the mathematical education research. Particularly, we show how some social factors in a determinate time (in this case the XVII century) influence on the development of series and function mathematical's concepts, and how an analysis of those events contribute to the didactical proposal's design for the study of those concepts in the class room.

Key words: Socioepistemological approach, series, function. 


\section{Introducción}

La importancia que se le ha conferido a la noción de serie en la matemática actual se ve reflejada en su presencia en el currículo de nivel superior, particularmente en el programa de Licenciatura en Matemáticas de la Universidad Pedagógica Nacional. Sin embargo, su estudio formal se realiza en semestres posteriores a aquellos en los que se presentan los espacios académicos Cálculo Diferencial e Integral, en los cuales consideramos que las series deberían constituir parte fundamental de las temáticas tratadas, puesto que tenemos como hipótesis que éstas intervienen en la evolución de conceptos como el de indivisible, infinitesimal, función, límite e integral.

Desde un punto de vista didáctico, en la historia se han determinado dos tendencias asociadas a la presentación y desarrollo del concepto matemático serie: una delineada desde los trabajos de Newton y sus contemporáneos y culminado con los de Lagrange, en el que las series son utilizadas en el marco de métodos heurísticos con el fin de dar solución a problemas propios de la época; en contraparte, la segunda tendencia surge a partir del trabajo de Cauchy, quien construyó el cálculo basado en el concepto de límite, el cual es fundamental en el estudio de series infinitas (Cantoral y Farfán, 1990). En este último, modelo la relación existente entre funciones y series se centra en el estudio de los polinomios de Taylor y de las series de Fourier.

Consideramos, al igual que Cantoral y Farfán (1990), que en los cursos de sucesiones y series es imperante el modelo proveniente de los trabajos de Cauchy, ya que uno de sus objetivos es estudiar la teoría formal de las series; esto puede implicar que se releguen los posibles tratamientos no formales (heurísticos) de dicho concepto, no se problematicen nociones inmersas en él, no se establezcan relaciones entre los conceptos o no se proporcione una significación de los mismos diferente a la que sugiere aquella tendencia.

Al adoptar el presupuesto de la aproximación socio-epistemológica de que todo conocimiento surge como construcción social y los conceptos presentaron una faceta operacional -correspondiente a la primera tendencia didáctica- antes de tener una estructural (Sfard, 1991), consideramos pertinente realizar una descripción de la primera faceta para el concepto de serie; en ese sentido, es de nuestro interés dar elementos para que en el futuro la primera tendencia didáctica propuesta por Cantoral sea tenida en cuenta para propuestas curriculares relacionadas con el cálculo, a partir de resaltar relaciones entre las series y otros conceptos como el de función o límite, de destacar en la historia la época en la cual tales conceptos tuvieron una faceta procedimental y de determinar con ayuda de la aproximación socio-epistemológica, problemas propios de un contexto en los que se muestre tal relación, métodos y resultados producto del tratamiento de los mismos, las concepciones que surgieron en medio de dicho tratamiento y la influencia de aspectos socio-culturales en todos esos procesos.

Presentamos entonces algunos de los referentes teóricos tenidos en cuenta para el desarrollo de este estudio, particularmente aquellos referidos a la aproximación socio-epistemológica; enseguida, con base en esa misma aproximación, explicitamos la metodología de 
estudio precisando las categorías que permitieron analizar la información; después, exponemos algunos resultados del estudio desarrollado, producto del análisis hecho; por último, presentamos algunas consideraciones finales en las cuales, entre otras cosas, sugerimos algunas tendencias de trabajo en el aula relativas a la temática ya referida.

\section{Algunos referentes teóricos}

El estudio realizado se enmarca en la aproximación socio-epistemológica desarrollada por Cantoral y Farfán (2004), quienes la proponen como un paradigma en la investigación en matemática educativa. Específicamente, coincidimos con estos autores en que es fundamental incorporar en la investigación aspectos pilares en la construcción social del conocimiento, como lo son (además de tales aspectos, dichos autores consideran otros dos: los planos cognitivos que involucra la construcción social del conocimiento y su transmisión mediante la enseñanza) su naturaleza epistemológica y su aspecto sociocultural con una aproximación en la cual dichos componentes se interrelacionan. Es, por tanto, una de las inquietudes de la aproximación socio-epistemológica, y también de nosotros, hacer que en la investigación en educación matemática se tengan presentes las prácticas sociales que han dado sentido a la matemática en un contexto particular, lo cual está en concordancia con lo expuesto por Sierpinska y Lerman en torno al papel de la epistemología en la educación matemática; para nuestro caso abordaremos tal aproximación en términos de la relación de las series con otros conceptos como el indivisible, infinitesimal, función, límite e integral.
En la aproximación socio-epistemológica se atiende a la construcción social del conocimiento, ya que su deseo es comprender las prácticas involucradas en un sistema didáctico sin dejar de lado el análisis de la transmisión de los saberes como modos culturales inmersos en un contexto social, es decir, despegándose de la visión de que estudiar matemáticas significa sólo apropiarse de nociones para acercarse a la idea de mirar a esta disciplina como una práctica social, y para comprender al hombre haciendo matemática en un tiempo, una sociedad y una cultura particulares. Además, se incorpora con mayor énfasis el componente epistemológico en sus investigaciones como una herramienta indispensable para la comprensión de los sucesos en un contexto particular y como una fuente de información respecto a las dificultades y modos de superación producidos en el desarrollo de las nociones o conceptos matemáticos, así como también de significados que, por los procesos de comunicación, se diluyen o pierden en el tiempo.

En ese sentido, y acorde con Farfán (idea presentada en una conferencia dada en la Escuela de Medicina, México, en mayo de 2000), consideramos que la aproximación socio-epistemológica es un marco para la investigación y el desarrollo del currículum que profundiza en el análisis del saber incorporando no sólo el origen conceptual o procedimental, sino su origen social. Una cierta razón de ser que es factible descubrir si se examinan las prácticas de referencia (prácticas que se caracterizan cuando se trate la construcción social del conocimiento matemático) y las formas de su aproximación en una cultura. En 
esencia, la socio-epistemología plantea (Cantoral, Molina y Sánchez, 2003) el examen del conocimiento social, histórica y culturalmente situado, problematizándolo a la luz de las circunstancias de su construcción y difusión.

En correspondencia con lo anterior, este estudio tiene como premisa ver la matemática no como los resultados de una actividad, sino como la actividad misma; no como un producto terminado, sino como un proceso que alberga tanto los resultados y las técnicas como los interrogantes, las conjeturas y los métodos que en una época determinada se plantearon para abordar la consideración de un determinado problema. Tenemos la intención de aprender y precisar el origen del conocimiento en relación con la influencia que tuvo el desarrollo de las series en la evolución del concepto de función, en términos de identificar los usos sociales que se le asocian desde su misma génesis, las concepciones, las dificultades y preguntas que provocaron su aparición, interrogantes y debates que produjeron controversias y que, a su vez, suscitaron su evolución en un contexto particular en el que las series tienen un estatus paramatemático (es decir, cuando el concepto fue utilizado como herramienta y no estudiado como objeto matemático).

Finalmente, pensamos que los acercamientos socioculturales dirigen sus esfuerzos a revelar la naturaleza epistemológica del saber, mediante una identificación de las circunstancias sociales y culturales que han permitido su consolidación; asimismo, identifica situaciones que contribuyeron al desarrollo conceptual de la ciencia. No se trata de investigación histórica, aunque se sirve de ella, sino que hay la intención de aprehender y precisar el origen del conocimiento, en términos de advertir algunos usos sociales, que se le asocian desde su misma génesis, los significados y los sentidos de los conceptos, evolución y desarrollo, al igual que su incursión en las instituciones educativas. Así, puede ser posible utilizar estos hallazgos para el diseño de situaciones en las cuales el saber se presente desde su propia epistemología.

\section{Construcción social del conocimiento matemático}

Un camino para alcanzar el objetivo propuesto, y que desarrollaremos en este trabajo, consiste en estudiar una comunidad específica de matemáticos que abordó temáticas relacionadas con las series y las funciones en el contexto mencionado: matemáticos europeos del siglo XVII en el marco de la solución del problema de cuadraturas. En dicho estudio, se pretende identificar algunas interacciones que se establecen entre los componente sociocultural, epistemología de las matemáticas y procesos cognitivos (en la componente cognitiva se destacan aspectos como la abstracción reflexiva, la generalización, el razonamiento bajo hipótesis, entre otros procesos), los cuales caracterizan, según Cantoral (2003, p. 1), la construcción social del conocimiento matemático.

Entenderemos por la componente sociocultural de la construcción social del conocimiento matemático a aquello que describe tanto los mecanismos mediante los cuales un medio específico y su cultura contribuyen a la formación del pensamiento matemático, como los métodos de validación y comunicación. Así, buscamos determinar la naturaleza sociocultural de los objetos matemáticos 
de interés, puesto que permite identificar los escenarios en los que éstos se significan o se usan.

Las interacciones, arriba referenciadas, propuestas por Cantoral (2003, p. 1) entre los componentes sociocultural, epistemología de las matemáticas y procesos cognitivos se denominan: memoria voluntaria y construcción social, matematización y construcción social, sociocultura y matemáticas, consenso y matemáticas y consenso y didáctica.

A nuestro parecer, estas interacciones constituyen el principal referente teórico de nuestro estudio, ya que delinearon el camino del mismo. Particularmente, las interacciones en las que estamos interesados para establecer algunos aspectos del origen y construcción social del conocimiento, en torno al concepto de serie, son sociocultura y matemáticas, consenso y matemáticas, matematización y construcción social, pues en ellas es relevante identificar los procesos que se siguieron para formalizar un conocimiento, los paradigmas imperantes en determinados contextos y en, consecuencia, la manera en que éstos afectaron la construcción y desarrollo de dicho conocimiento, y cuáles eran los mecanismos de consenso que permitían guiar la actividad de los grupos sociales ante un tipo de situaciones.

Observamos que a partir de la anterior descripción sobre la socio-epistemología como aproximación teórica, la construcción social del conocimiento matemático se caracteriza en términos de tres interacciones específicas, las cuales adoptaremos en este trabajo como las categorías de análisis de información. En este sentido, compartimos la postura de Castañeda (2004, p. 39) sobre tal aproximación teórica como una metodología para el análisis pretendido.

\section{Método y metodología del estudio}

El estudio aquí reportado es de tipo cualitativo descriptivo, ya que en primera instancia, analizamos los datos mediante la reunión, clasificación, ordenación, síntesis e interpretación; además, establecimos prácticas o puntos de vista que prevalecieron y condiciones que las propiciaron, entre otros aspectos.

Por otro lado, como método (entenderemos por método la gama de aproximaciones empleadas en la investigación educativa, para reunir los datos que van a emplearse como base para la inferencia y la interpretación, para la explicación y la predicción. En síntesis, son las técnicas más generales que emplearemos en esta investigación) de investigación empleamos una aproximación al método histórico en investigación educativa. Para el desarrollo de este método, Cohen y Manion (1990) sugieren las siguientes fases: limitación de un problema o de un área de estudio, formulación de unas hipótesis o conjunto de cuestiones (algunas veces), reunión, organización, validación (o evaluación), selección y análisis de los datos, prueba de las hipótesis o contestación de las cuestiones (cuando corresponda) y, finalmente, la redacción de un informe de investigación, expuesto por los mismos autores, puesto que este último permitió interpretar una situación del pasado (ya sea utilizando la evidencia existente o interpretaciones de tal evidencia) con el fin de identificar hechos y extraer conclusiones acerca de acontecimientos ocurridos, mediante el análisis de los datos recogidos. Específicamente para llevar a cabo el análisis 
de los datos, usamos la aproximación socio-epistemológica en su faceta metodológica.

\section{Categorías de análisis}

Para el análisis de datos, como mencionamos en la descripción de la construcción social del conocimiento, utilizaremos la aproximación socio-epistemológica como metodología que permitirá precisar las categorías de análisis. Particularmente, como ya comentamos, las interacciones en las que estamos interesados para establecer las categorías de análisis son: sociocultura y matemáticas, consenso y matemáticas, y matematización y construcción social. A continuación caracterizaremos cada una de tales interacciones.

\section{Sociocultura y matemática}

Atendiendo a que las matemáticas son una construcción social y, por tanto, están enmarcadas en contextos particulares, este tipo de interacción da cuenta de las prácticas sociales de referencia imperantes en tales contextos y, en consecuencia, cómo éstas afectaron la construcción de dicho conocimiento, permitiendo la aparición de concepciones y, por ende, la resignificación (es decir, la posibilidad de enriquecer o cambiar significados de los conocimientos) de los conceptos inmersos en esa construcción, además de "ratificar la hipótesis de que los conceptos matemáticos son primero usados antes de ser definidos" (Martínez, 2003, p. 35). Una descripción específica de prácticas sociales de referencia es:

Las prácticas sociales de referencia son aquellas acciones intencionales que un grupo humano específico realiza, influenciado por el contexto social y cultural en que éste se encuentra, con el objetivo de transformar, complementar y desarrollar un conocimiento. En ningún momento estas prácticas tienen la intención de describir la situación particular de un individuo, sino los contextos que son comunes a un grupo [...] (Cantoral, 2003, p. 7).

Esta descripción de práctica social de referencia nos sugiere, además, tener en cuenta la influencia de ciertas instituciones o circunstancias (políticas, económicas, religiosas) en la construcción de un conocimiento.

\section{Consenso y matemáticas}

En la construcción del conocimiento se articulan mecanismos de consenso que permiten guiar la actividad de los grupos sociales ante cierto tipo de situaciones, lo cual hace previsible su desempeño en condiciones similares. Dichos mecanismos se basan en la comunicación del conocimiento.

Entenderemos consenso como la herramienta que permite, por un lado, explicar el surgimiento de prácticas compartidas que establecen: cuándo un conocimiento es válido $\mathrm{y}$, por otro, describir las prácticas existentes.

Con base en lo anterior, consideramos que es importante tener en cuenta algunos factores sociales, puesto que ellos influyen a los individuos creando restricciones y no permitiendo que por voluntad individual se establezca un consenso; por ejemplo, se puede establecer que un factor de este tipo lo constituyó la iglesia ya que influenció los trabajos científicos.

Como un aspecto importante en el consenso es la comunicación, concebi- 
mos este término en el mismo sentido que lo propone Castañeda (2004, p. 3):

Los procedimientos por los que se "expresan las ideas" y se reproducen "ideologías" o "formas de pensar". La comunicación se logra a través de un lenguaje que comparten un grupo de personas, de acuerdo con la información de que pueden disponer, con su nivel de tecnología. En el caso de la comunicación escrita, se utiliza un conjunto de símbolos cuya codificación se comparte.

\section{Matematización y construcción social}

Consideramos que existen dos tipos de matematización en un contexto específico; por un lado, se generan modelos matemáticos que permitan describir o tratar ciertas situaciones y, por el otro, existe el modelo y se acopla la situación a éste para ser tratada. Así, los aspectos socioculturales (específicamente las prácticas sociales de referencia -paradigmas de pensamiento-, mecanismos de consenso y comunicación), inmersos en el contexto que deseamos estudiar, permiten identificar algunos procesos de matematización que contribuyeron al propio desarrollo de la matemática.

Como se puede inferir de las anteriores interacciones y de lo dicho acerca de éstas, los aspectos que caracterizan a las dos primeras de ellas tienen una influencia directa en esta última, pero, a su vez, la interacción sociocultura y matemáticas influye en la interacción que refiere al consenso por cuanto los procesos que allí se llevan a cabo conllevan procesos de validación.

\section{Algunos resultados del estudio}

Para llevar a cabo el estudio, fue necesario realizar un recuento histórico, el cual nos permitió identificar diferentes aspectos acerca de la relación de las series con los conceptos de función o límite. Uno de tales aspectos se enmarca en el uso que se les daba a las primeras (sin ser estudiadas como tal) a finales del siglo XVII, principalmente para realizar cálculos referentes a cuadraturas y cubaturas por medio de métodos heurísticos (considerados novedosos para tal época), que rescataban tratamientos trabajados anteriormente por Arquímedes y basados, a su vez, en la corriente atomista propuesta por Demócrito y retomada en aquella época por Cavalieri. Así, identificamos al siglo XVII como parte de la época en la cual las series se caracterizaron como objeto paramatemático, puesto que en él observamos una proliferación de tales métodos y de personajes que trabajaron al respecto como Cavalieri, Roberval, Fermat, Pascal, Wallis, Gregory de St. Vincent, Newton, James Gregory y Leibniz. Particularmente, abordamos el problema de cuadraturas (este problema se centra en establecer el área de una superficie; en particular los matemáticos griegos abordaron dicha problemática intentando hacer corresponder tal área con la de la región interior que define un cuadrado. A este procedimiento se le llamó cuadratura. En la actualidad, se asocia al término cuadratura el cálculo de áreas, sin hacer alusión a la relación de ésta con algún cuadrado) de las parábolas generalizadas (las parábolas generalizadas son aquellas cuya expresión analítica es $y=k x^{n}$ con $n$ un número racional diferente de -1 y $k$ un número real), representativo de la época -otros problemas abordados en ella son el estudio de tangentes, máximos y mínimos-, y bajo éste hicimos el estudio de las contribuciones de los autores men- 
cionados, matemáticos como Hermann, los Bernoulli y Euler afirmaron que estos personajes fueron los fundadores de los métodos del nuevo cálculo y que, por tanto, merecían y debían ser estudiados; pues en sus trabajos aparece el uso de infinitesimales, el trabajo con series, la representación de funciones por medio de series, la utilización de la geometría analítica, la aritmetización de la geometría, el uso de la simbología algebraica, la aparición de la noción de límite y de convergencia, etc., con el objeto de establecer relaciones entre las series con otros conceptos.

A continuación, presentamos un ejemplo de cómo un aspecto sociocultural de la época influyó en un cambio de pensamiento que se ve reflejado en los métodos utilizados por algunos matemáticos para calcular cuadraturas; este ejemplo constituye un resultado del análisis de la información elaborado bajo las categorías de análisis ya referenciadas. Como muestra de tal análisis se ilustran algunas consecuencias de dichos cambios de pensamiento en la construcción del conocimiento matemático y algunas concepciones de los conceptos de interés encontradas en la época, estipulando las condiciones de comunicación y validación bajo las cuales se presentaron.

\section{Influencia sociocultural: el método experimental, un cambio de pensamiento}

Para justificar por qué en este siglo hubo una proliferación de métodos heurísticos novedosos para la solución del problema de cuadraturas (y en general de todos los problemas de interés para la comunidad de matemáticos), se hizo- pertinente tener en cuenta el contexto social europeo de aquella época y, en esencia, varios aspectos socioculturales -prácticas sociales de referencia- entre los cuales destacamos la revolución científica característica del siglo XVII. Esta revolución trajo como consecuencia un cambio de pensamiento a raíz de la propuesta de Bacon (1561-1626), quien cuestionó las bases del conocimiento aristotélico en su obra Novum Organum (1620), mostrando explícitamente los prejuicios del pensamiento dogmático y convencional y estableciendo las bases del método experimental basado en la inducción científica, caracterizada porque los conceptos generales y el conocimiento de la naturaleza surgen de la reiteración de observaciones de hechos particulares, analizados de manera sistemática. El pensamiento baconiano adoptado por los científicos constituyó la base para obtener varios y fructíferos resultados no previsibles hasta el momento y trajo como consecuencia un lugar preferente para el investigador experimental en la sociedad de éste y de los siglos siguientes. Al respecto, Cohen y Manion (1990, p. 26) exponen que: La historia del razonamiento iba a sufrir un cambio dramático en el siglo XVII, cuando Francis Bacon empezó a poner un creciente énfasis sobre la ciencia basada en la observación. Al criticar el modelo del razonamiento deductivo sobre la base de que sus premisas mayores a menudo eran nociones preconcebidas que polarizaban inevitablemente las conclusiones, propuso en su lugar el método del razonamiento inductivo, por medio del cual el estudio de un número de casos individuales debiera llevar a una hipótesis y finalmente a una generalización. [...] La mayor contri- 
bución de Bacon a la ciencia fue tal, que fue capaz de rescatarla del punto muerto del método deductivo, cuyos abusos habían llevado a la detención del proceso científico.

\section{Tres métodos para el cálculo de cuadraturas: evidencia de un cambio de pensamiento}

A continuación presentamos tres métodos de cálculo de cuadraturas propuestos en el siglo XVII, uno realizado por Cavalieri y los otros dos realizado por Fermat, mediante los cuales pretendemos hacer evidentes, entre otras cosas, el cambio de pensamiento antes referenciado entre un método y otro.

\section{Método de i}

Cavalieri, para encontrar el área del segmento parabólico determinado por la curva ya mencionada, y el área de otros (aunque Barrios (1995, p. 320) dice que Cavalieri hizo un proceso análogo para determinar el área de los segmentos de parábola para $n=1, \ldots, 6,9$, no hemos encontrado en la literatura revisada el desarrollo para dichos casos) que están determinados bajo la curva general $y$ $=x^{n}$, denota a la colección de todos las líneas o indivisibles, en este caso segmentos paralelos entre sí, de una figura plana como $T L(F)$; y en el mismo sentido denota $T C(F)$ o $T L^{2}(F)$ a la colección de todos los cuadrados paralelos y semejantes entre sí (indivisibles), construidos sobre cada una de las líneas de $F$.

Cavalieri está interesado en encontrar la relación existente entre dos colecciones; por ejemplo, para encontrar el área del segmento parabólico determinado por la curva $y=x^{1}$, divide el rectángulo $A B C D$ en dos triángulos a partir del segmento $A C$ (Figura 1). Enseguida toma $C G=A F$ y a cada indivisible $E F$ del triángulo $A B C$ le hace corresponder el indivisible $H G$ del triángulo $C D A$; de lo anterior, deduce que $E F=H G$ y, por ende, que $T L(A B C)$ es igual a $T L(C D A)$, con lo que deduce que la razón (en este caso podemos notar de manera explícita que aunque no se hace uso de medidas, sí se evidencia la razón a partir de la construcción misma) entre $T L(A B C D)$ y $T L(A B C)$ es $2: 1$.

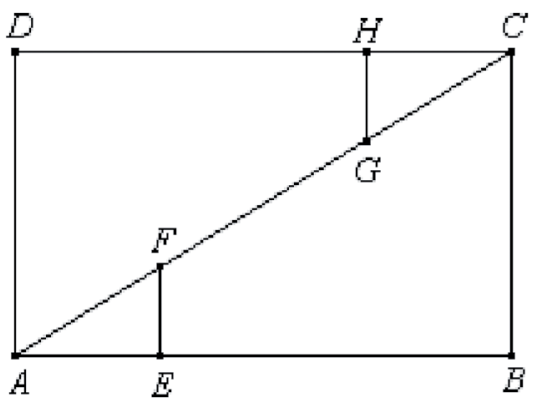

Figura 1

Ahora, para el segmento parabólico determinado por $y=x^{2}$, Cavalieri construye cuadrados sobre los indivisibles (segmentos paralelos al segmento $A D$ ) del rectángulo $A B C D$ y sobre los indivisibles (segmentos paralelos al segmento $C B$ ) del triángulo $A B C$ (Figura 2). Sea el segmento $R V$ un indivisible de $A B C D, F$ y $E$ los puntos medios de los segmentos $A D$ y $B C, G$ y $M$ los puntos medios de los segmentos $A B$ y $F E$, y $S$ y $T$ los puntos de corte de $R V$ con $F E$ y $A C$, respectivamente. 


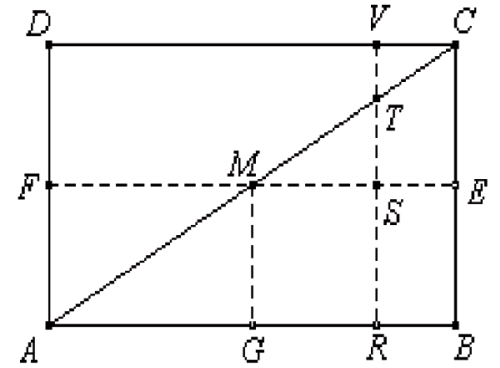

Figura 2

Bajo estas condiciones y a partir del cuadrado construido sobre el indivisible $R V$ (Figura 3), se asegura que $R T^{2}+T V^{2}$ $=2\left(R S^{2}+S T^{2}\right)$.

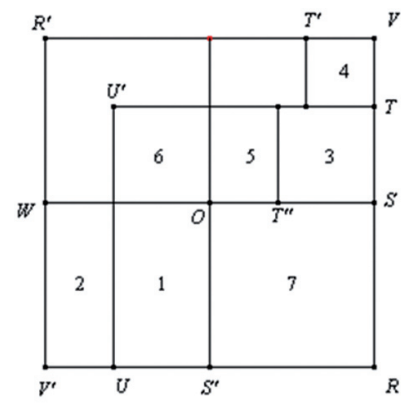

Figura 3

Tal resultado se obtiene de considerar el cuadrado de lado $R V$, en el que $R V$ $=R V, R S=R S^{\prime}, T V=T^{\prime} V, T S=T^{\prime \prime} S, U S^{\prime}=$ $S T$ y $R T=R U$. Con ello, se tiene que $R T^{2}$ $=1+3+5+6+7$ (lo que significa que el cuadrado $R T U$ 'U está conformado por las regiones, debe tenerse en cuenta que los números representan las regiones en las que éstos están centrados; por ejemplo, el número 7 representa la región limitada por el cuadrado OSRS'- 1, 3, 5, 6 у 7$), T V^{2}=4,2 R S^{2}=2+1+7$ y $2 S T^{2}$
$=6+3$; ahora, como $2=4+5$, puesto que $T V=V^{\prime} U, V^{\prime} W=S V$ y $O T^{\prime \prime}=V T$, se concluye que

$R T^{2}+T V^{2}=(1+3+5+6+7)+4=(2$ $+1+7)+(3+6)=2 R S^{2}+2 S T^{2}=2\left(R S^{2}\right.$ $\left.+S T^{2}\right)$.

Pero como esto ocurre para todos los indivisibles $R V$ de $A B C D$, Cavalieri sostiene que las correspondientes colecciones de cuadrados guardan la misma relación, es decir:

$T C(A B C)+T C(A C D)=2 T C(A B E F)+$ $2 T C(M E C)+2 T C(A M F)$

'Puesto que $T C(A B C)$ se refiere a la colección de cuadrados de todos los $R V$, $T C(A C D)$ a la de todos los $T V, 2 T C(A B E F)$ a la de dos veces la colección de cuadrados de todos los $R S$ y $2 T C(M E C)+$ $2 T C(A M F)$ a la de dos veces la colección de cuadrados de todos los ST.

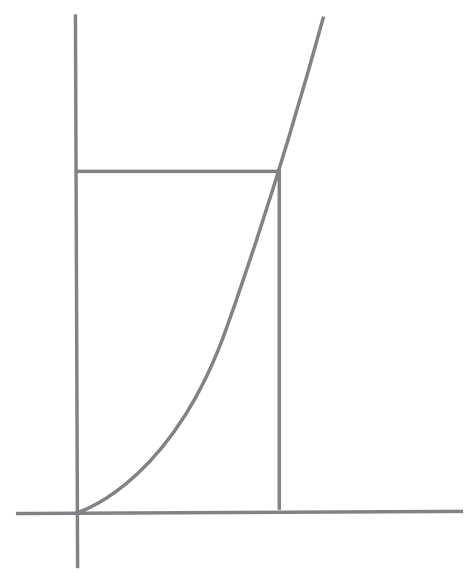

Figura 4 
De ahí, como $T C(A B C)=T C(A C D) \mathrm{y}$ $T C(M E C)=T C(A M F)$, entonces

1) $T C(A B C)=T C(A B E F)+2 T C(A M F)$

Por otra parte, se puede deducir que

2) $T C(A B C D)=4 T C(A B E F)$

$\mathrm{Y}$

$T C(A B C D)=8 T C(A G M F)$

Ahora, Cavalieri, utilizando su teorema la razón entre todos los cuadrados de un paralelogramo y todos los cuadrados de uno de sus triángulos diagonales es constante, se obtiene: para este caso particular que $T C(A B C D)$ : $T C(A B C)=$ $T C(A G M F): T C(A M F)$ y como $T C(A B C D)$ $=8 T C(A G M F)$, entonces $T C(A B C)=8$ TC $(A M F)$,

\section{3) $T C(A B C)=8 T C(A M F)$}

De 1), 2) y 3) se obtiene que $T C(A B C D)=4 T C(A B E F)$

$$
\begin{aligned}
& =4 T C(A B C)-8 T C(A M F) \\
& =4 T C(A B C)-1 T C(A B C) \\
& =3 T C(A B C)
\end{aligned}
$$

Luego, la razón entre $T C(A B C D)$ y $T C(A B C)$ es 3:1.

\section{Métodos de Fermat} Método uno

Fermat fue influenciado por Diofanto (200/214-284/298) en la teoría de números, por Apolonio (262-190 a. C.), Pappus (siglo III-IV) y François Viète (1540-1603), en su creación de una geometría analítica, y por Arquímedes y Cavalieri en su tratamiento de cuadraturas; en relación con éstas y particularmente con el resultado obtenido por Cavalieri para $\int_{0}^{a} x^{n} d x$. Fermat, de manera análoga a Cavalieri, presenta el resultado del cálculo de cuadraturas de parábolas como razones entre áreas; sin embargo, consideramos que estas razones se establecen en términos de las medidas de las magnitudes -más que en las magnitudes mismas, como lo hacía Cavalieri- (áreas) del segmento parabólico y del rectángulo circunscrito a él, tal como lo muestra la Figura 4.

Concretamente, el resultado obtenido por él fue la razón $\frac{1}{k+1}$, al utilizar argumentos similares a los que actualmente se escriben como

$$
\lim _{n \rightarrow \infty} \frac{1^{k}+2^{k}+3^{k}+\ldots+n^{k}}{n\left(n^{k}\right)}=\frac{1}{k+1}
$$

Para ello, Fermat utiliza las sumas:

$$
\begin{gathered}
\sum_{i=1}^{n} \frac{i(i+1)}{2 !}=\frac{n(n+1)(n+2)}{(2+1) !} \\
\sum_{i=1}^{n} \frac{i(i+1)(i+2)}{3 !}=\frac{n(n+1)(n+2)(n+3)}{(3+1) !}
\end{gathered}
$$

Para expresar de manera general (fórmula expresada en una carta enviada a Mersenne en 1636, sin prueba), a partir del método de inducción incompleta, que

$$
\text { 4) } \quad \sum_{i=1}^{n} \frac{i(i+1) \ldots(i+k-1)}{k !}=\frac{n(n+1)(n+2) \ldots(n+k)}{(k+1) !}
$$


Él desarrolla el producto $i(i+1)(i+$ $2) \ldots(i+k-1)$ y obtiene una expresión de la forma $i^{k}+a_{1} i^{k-1}+\ldots+a_{k-1} i$, que utiliza para representar:

$$
\begin{aligned}
& \sum_{i=1}^{n} \frac{i(i+1) \ldots(i+k-1)}{k !} \\
& \text { Como } \\
& \frac{1}{k !}\left[\sum_{i=1}^{n} i^{k}+a_{1} \sum_{i=1}^{n} i^{k-1}+\ldots+a_{k-1} \sum_{i=1}^{n} i\right]
\end{aligned}
$$

Con lo que por 4) se obtiene:

$$
\frac{1}{k !}\left[\sum_{i=1}^{n} i^{k}+a_{1} \sum_{i=1}^{n} i^{k-1}+\ldots+a_{k-1} \sum_{i=1}^{n} i\right]=\frac{n(n+1)(n+2) \ldots(n+k)}{(k+1) !}
$$

De lo cual:

5) $\sum_{i=1}^{n} i^{k}=\frac{n(n+1)(n+2) \ldots(n+k)}{k+1}-\left[a_{1} \sum_{i=1}^{n} i^{k-1}+\ldots+a_{k-1} \sum_{i=1}^{n} i\right]$

Que corresponde a una fórmula por recurrencia para calcular la suma de las $k$-ésimas potencias de los $n$ primeros números naturales. Así, para $k=2$ tenemos que

$$
\sum_{i=1}^{n} i^{2}=\frac{n(n+1)(n+2)}{2+1}-\sum_{i=i}^{n} i
$$

Y como

$$
\sum_{i=1}^{n} i=\frac{n(n+1)}{2}=\frac{n^{2}}{2}+\frac{n}{2}
$$

Entonces:

$\sum_{i=1}^{n} i^{2}=\frac{n(n+1)(2 n+1)}{6}=\frac{2 n^{3}}{6}+\frac{3 n^{2}}{6}+\frac{n}{6}=\frac{n^{3}}{3}+\frac{n^{2}}{2}+\frac{n}{6}$

A partir de lo anterior y de la fórmula 5) se obtiene que

$$
\sum_{i=1}^{n} i^{k}=\frac{n^{k+1}}{k+1}+b_{1} \frac{n^{k}}{k+1}+b_{2} \frac{n^{k-1}}{k+1}+\cdots+b_{k} \frac{n}{k+1}
$$

De 5) podemos observar que:

$$
\begin{aligned}
& \frac{\sum_{i=1}^{n} i^{k}}{n\left(n^{k}\right)}=\frac{\frac{n^{k+1}}{k+1}+b_{1} \frac{n^{k}}{k+1}+b_{2} \frac{n^{k-1}}{k+1}+\cdots+b_{k} \frac{n}{k+1}}{n\left(n^{k}\right)} \\
& =\frac{1}{k+1}+\frac{b_{1}}{n(k+1)}+\frac{b_{2}}{n^{2}(k+1)}+\cdots+\frac{b_{k}}{n^{k}(k+1)}
\end{aligned}
$$

Pero si $n$ es muy grande, entonces cada uno de los sumandos con numerador $b_{i}$ tiende a cero; actualmente esta razón podemos escribirla como

$$
\lim _{n \rightarrow \infty} \frac{\sum_{i=1}^{n} i^{k}}{n\left(n^{k}\right)}=\frac{1}{k+1}
$$

Resaltamos que $\sum_{i=1}^{n} i^{k}$ puede interpretarse en términos de Cavalieri, como $T L^{k}(F)$, y $n\left(n^{k}\right)$ como $\operatorname{TL}^{k}(R)$, en la cual $F$ representa el segmento parabólico determinado por $y=x^{k}$ y $R$ el rectángulo en el que $F$ está inscrita.

\section{Método dos}

Para encontrar la medida del área bajo la curva $y=k x^{n}$, con $n$ racional distinto de -1 , en el intervalo [0, a], en términos actuales, Fermat dividió dicho intervalo en una cantidad infinita de subintervalos, de tal forma que los puntos de dicha división conformaran la progresión geométrica decreciente con términos, $a, a r, a r^{2}, a r^{3}, \ldots, a r^{n}, \ldots$, lo que significa, para este caso, que la razón $r$ es menor que 1; enseguida estableció las ordenadas para cada uno de estos puntos, lo 
cual le permitió construir rectángulos circunscritos para aproximar la medida del área que deseaba buscar. Tal aproximación la hizo a partir de la suma de la medida del área de cada uno de dichos rectángulos (Figura 5).

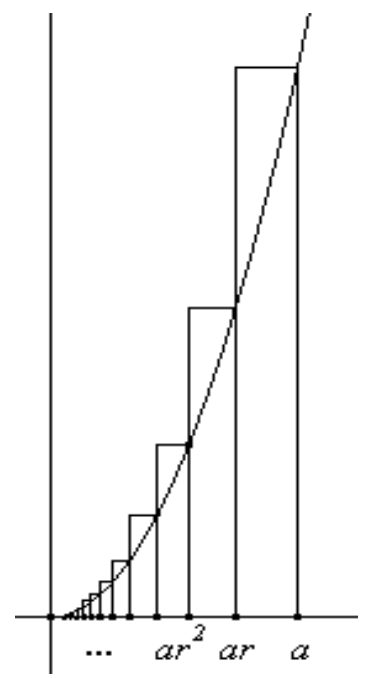

Figura 5

Como la medida del área de cada uno de los rectángulos está dada por $(a-a r)$ $k a^{n},\left(a r-a r^{2}\right) k(a r)^{n},\left(a r^{2}-a r^{3}\right) k\left(a r^{2}\right)^{n}, \ldots$, $\left(a r^{n}-a r^{n+1}\right) k\left(a r^{n}\right)^{n}, \ldots$, respectivamente, la suma de éstas se determina a partir de la serie geométrica:

$$
\begin{aligned}
& \mathrm{S}=(a-a r) k a^{n}+\left(a r-a r^{2}\right) k(a r)^{n}+\left(a r^{2}\right. \\
& \left.-a r^{3}\right) k\left(a r^{2}\right)^{n}+\ldots+\left(a r^{n}-a r^{n+1}\right) k\left(a r^{n}\right)^{n}+\ldots \\
& \mathrm{S}=a(1-r) k a^{n}+a r(1-r) k(a r)^{n}+a r^{2}(1 \\
& -r) k\left(a r^{2}\right)^{n}+\ldots+a r^{n}(1-r) k\left(a r^{n}\right)^{n}+\ldots \\
& \mathrm{S}=\mathrm{a}(1-\mathrm{r}) \mathrm{ka}^{n}\left(1+\mathrm{r}^{n+1}+\mathrm{r}^{2 \times n+2}+\ldots+\right. \\
& \mathrm{r} \begin{array}{l}
n \times n+n \\
+\ldots)
\end{array}
\end{aligned}
$$

Fermat, con base en su propiedad para la suma de progresiones geométricas, obtuvo:

$$
\frac{1-r^{n+1}}{r^{n+1}}=\frac{1}{S_{1}-1}
$$

En la cual:

$S_{1}=1+r^{n+1}+r^{2 n+2}+\ldots+r^{n \cdot n+n}+\ldots$

Luego:

$$
S_{1}=\frac{r^{n+1}}{1-r^{n+1}}+1=\frac{r^{n+1}+1-r^{n+1}}{1-r^{n+1}}=\frac{1}{1-r^{n+1}}
$$

Así,

$$
S=\frac{(1-r) k a^{n+1}}{1-r^{n+1}}
$$

Pero:

$$
S=\frac{(1-r) k a^{n+1}}{1-r^{n+1}}=\frac{k a^{n+1}}{\frac{1-r^{n+1}}{1-r}}=\frac{k a^{n+1}}{1+r+r^{2}+\ldots+r^{n}}
$$

De lo anterior, podemos interpretar que Fermat permite que $r$ tienda a 1 , con lo cual las bases de los rectángulos circunscritos se comprimen hacia $a$, por lo que la aproximación por dichos rectángulos es más fiel al valor del área buscada. Con la escritura actual:

$$
\lim _{r \rightarrow 1} \frac{k a^{n+1}}{1+r+r^{2}+\ldots+r^{n}}=\frac{k a^{n+1}}{n+1}
$$

En los anteriores métodos, podemos evidenciar las consecuencias del cambio de pensamiento causado por Bacon. Por un lado, Cavalieri aún conserva en su método un enfoque geométrico que venía desde la época de Arquímedes y que, por tanto, buscaba cierta rigurosidad en 
la justificación y presentación de resultados (dogmático, deductivo y sin asociar números a las áreas calculadas); por otro, Fermat, influenciado un poco más por la propuesta baconiana de inducción científica, propuso métodos aritméticoalgebraicos, dando inicio así al cálculo de infinitesimales y, por ende, a lo que se ha denominado la aritmetización del cálculo. En él se vislumbra la esencia de la llamada inducción incompleta (especialmente en su primer método) realizada por matemáticos de la época con el objetivo de encontrar resultados de manera eficiente y eficaz, a partir de regularidades y generalizaciones, sin caer en la rigurosidad que se tenía con el paradigma antiguo.

\section{Algunos consensos y aspectos de la construcción social del conocimiento}

En los tratamientos de los diversos personajes de la época, pese a diferencias muy particulares, como la presentada anteriormente en relación con Cavalieri y Fermat, se resalta que desde un punto de vista conceptual hubo ciertos consensos; por ejemplo, todos entienden un indivisible como la unidad mínima que compone un cuerpo y que, además, infinitos indivisibles conforman a dicho cuerpo. En este sentido, dichos personajes entendieron un indivisible como un infinitesimal, en tanto es una cantidad infinitamente pequeña, no obstante no la consideraban variable, como sí lo hizo Cauchy más tarde, sino como cantidades aritméticas que se podían manipular como tal (ese es el caso de Fermat, Wallis, Roberval, etc.). Ahora bien, consideramos pertinente resaltar que, aunque Fermat y Cavalieri entienden un indivisible de la misma manera, en dichos procedimientos se observa una caracterización de ellos cualitativamente diferente en términos de la dimensionalidad; por ejemplo, para encontrar áreas, Cavalieri utiliza indivisibles que tienen una magnitud de menor dimensión que la de la magnitud del cuerpo (i. e., segmentos para calcular áreas de superficie), mientras que Fermat, en particular en su segundo método, utiliza indivisibles que tienen una magnitud de igual dimensión que la de la magnitud del cuerpo. Ello permite establecer que en los trabajos de Cavalieri se presenta el denominado problema de heterogeneidad de dimensiones (cabe resaltar que Roberval ya había advertido el problema de heterogeneidad presente en los trabajos de Cavalieri, y que desde él podemos decir que hubo un consenso al respecto en términos de tener presente la misma dimensionalidad de los indivisibles a la hora de calcular el área (o el volumen) de alguna superficie, o cuerpo). En contraste, el método de Fermat no lo evidencia y, por el contrario, se convierte en un antecedente directo al método para el cálculo de una integral definida de Riemann, puesto que utiliza rectángulos infinitesimales (convenientemente construidos) y encuentra su área, para luego sumarlas con el objetivo de lograr la mejor aproximación posible al área del segmento parabólico. Queremos resaltar también que, en relación con el concepto de serie, por primera vez un matemático (Fermat) de aquella época usa este objeto de manera explícita para encontrar dicha aproximación y para expresar, indirectamente, a

$$
\frac{1}{1-r^{n+1}}
$$

(una función racional de variable $\mathrm{r}$ ) con la serie geométrica $1+r n+1+r 2 n+2$ $+\ldots+\mathrm{r} n . \mathrm{n}+\mathrm{n}+\ldots$, la cual construyó 
de tal forma que la razón $(r n+1)$ fuese menor que uno, lo que permite notar un uso tácito de la condición (actual) de convergencia para series geométricas, a saber: una serie geométrica con razón s converge si $|\mathrm{s}|<1$. Este método de aproximar áreas por medio del uso de series fue reiterativo en trabajos de personajes contemporáneos o posteriores a Fermat como Wallis, Newton y Leibniz.

A partir tanto de la evolución de la simbolización, como de la geometría analítica impulsada por Fermat y Descartes, la aritmetización y algebrización de los métodos de cuadraturas, similares a los de Fermat, permitió otro consenso entre los matemáticos, en términos de combinar representaciones aritméticas, algebraicas y gráficas para la obtención de resultados de manera heurística, dejando de lado aquellos métodos geométricos que precisaban de justificaciones rigurosas.

Ahora bien, queremos resaltar que, tal como presupone el referente teórico y metodológico, la comunicación es uno de los medios mediante el cual un conocimiento puede validarse, difundirse $y$, por ende, lograr consensos entre los miembros de una comunidad; vemos, por ejemplo, que gracias a la consolidación de mecanismos de comunicación (personal, por correspondencia o por comunicadores (para la comunidad de científicos existieron personajes que se encargaron de esa labor -informadores profesionales- como Mersenne, Wallis, Boyle, Oldenburg y Huyghens, quienes facilitaban la comunicación entre científicos; John Collins (1625-1683), por ejemplo, recopiló correspondencia que trataba hechos científicos de la época en un libro publicado en 1712 y titulado (Commercium epistolicum) que se presentaron en la segunda mitad del siglo XVII, los personajes mencionados tuvieron la posibilidad de conocer y dar a conocer trabajos con mayor rapidez, logrando así una difusión de conocimiento y la posibilidad de establecer una discusión en torno a éste. Es de resaltar que en esta época los medios de transporte y de comunicación mejoraron considerablemente, creando así oficinas de diligenciamiento y de correspondencia privada (ya existía la correspondencia de carta de Estado) en Inglaterra, pero que se fueron extendiendo con cierta rapidez en Europa; a esto se debe aunar el hecho de que tanto el intercambio comercial como la influencia de la Iglesia fortalecieron, de manera indirecta, el intercambio de pensamientos. Tengamos presente, por ejemplo, que la persecución a ciertos miembros de la comunidad de científicos (entre ellos matemáticos como Descartes) por parte de la Iglesia, no obstante el estancamiento que en principio causó al desarrollo del conocimiento, hizo que éstos se desplazaran o bien a países que no presentaban obstáculos para el desarrollo de conocimientos o a centros creados de manera privada por reyes o personajes que estaban interesados en dicho desarrollo. Así, por ejemplo, en Francia, se crearon las academias Collège Royal, la Académie Parisienne y la Académie des Sciences. Por otro lado, esta limitación trajo consigo la creación de nuevas doctrinas religiosas, las cuales dieron cabida al estudio de la naciente ciencia y también a la creación de academias que pretendían difundir este nuevo conocimiento. 


\section{Consideraciones finales}

A partir del análisis realizado bajo la aproximación socio-epistemológica, se hace evidente que realmente los aspectos socioculturales de un contexto particular influyen en la construcción del conocimiento. Específicamente en la época que nos interesó identificamos varios de tales aspectos, unos que muestran algunas prácticas sociales de referencia (e. g., el cambio de pensamiento originado por la propuesta baconiana) y otros, la intervención de instituciones externas que intervienen en dicha construcción (e. g., la influencia de la Iglesia). Precisamos que a partir de tales prácticas sociales surgieron nociones de conceptos que estuvieron sujetas a las mismas y que influyeron en el desarrollo de los conceptos de serie y función; por ejemplo:

- Surgieron diversas interpretaciones del concepto de indivisible que, aunque fue considerado por todos los personajes de la época como la mínima componente que conforma un cuerpo, presentó diferencias acerca de la dimensión que éste podía tener con respecto a la del cuerpo que conforma; es importante tener en cuenta que para Cavalieri, Roberval, Fermat, Pascal y Wallis, los términos indivisible e infinitesimal podían ser utilizados indistintamente, aunque algunos los consideraron desde un punto de vista geométrico (Cavalieri, Roberval), mientras que otros, basados en la geometría, les daban un tratamiento aritmético (Roberval, Fermat, Wallis, Newton). Además, a diferencia de la propuesta de Cauchy (que consideraba los infinitesimales como variables cuyo límite es cero), en el siglo XVII por lo general el indivisible y el infinitesimal eran tratados más como una constante que como una variable.
- Identificamos las siguientes concepciones de serie en su estatus paramatemático:

- Como herramienta, puesto que se utiliza como representación de una expresión (bien sea número o función) en los procedimientos para calcular cuadraturas. Además en esta concepción hay una de aproximación, ya que las series aparecen (de manera explícita o implícita) en tales procedimientos. Un ejemplo claro de lo anterior puede verse para el caso de Fermat.

- Como producto, ya que de manera explícita el resultado del cálculo de la cuadratura de una función se representa mediante una serie, o lo que es representado en series es la función misma.

- Como representación. En cualquiera de los dos casos anteriores vemos cómo una expresión puede ser representada mediante una serie de potencias.

- Observamos la presencia de la concepción de función como curva (analíticogeométrica) desde los trabajos de Roberval, Fermat, Wallis y Newton (en tanto surge la geometría analítica) y, en particular, cómo se dio un cambio hacia la concepción de función como expresión analítica, a partir del trabajo con series elaborado específicamente por Newton, quien admitió de manera explícita que una expresión podía ser representada por una serie de potencias. El estudio realizado nos permitió identificar que las series influyeron en el desarrollo del concepto de función en tanto suscitaron el tránsito entre la concepción de función como curva a la de función como expresión analítica, puesto que el uso de las series para el cálculo de cuadraturas, en primera instancia, sugirió la aproximación de valores reales, para luego permitir la representación de expresiones mediante series.

A partir de los resultados que en parte hemos presentado en este reporte, sugerimos algunas tendencias que pue- 
den ser tenidas en cuenta a la hora de estudiar funciones o algunos aspectos del cálculo integral, teniendo como base el uso de las series como herramienta más que como un concepto estructurado, rescatando concepciones de serie que tienen que ver con la aproximación y con la representación, pero, además, nociones de otros conceptos que están inmersos en este estudio, como el de límite, convergencia, infinitesimal, etc., que muchas veces permanecen ocultos y que, por tanto, no son problematizados, hecho que conduce a que éstos no adquieran un significado para el estudiante.

Una de dichas tendencias consiste en abordar el problema de cuadraturas, el cual, como se mostró con algunos ejemplos, permite problematizar todos los conceptos anteriormente mencionados y sugerir temáticas de propuestas que se centren en el estudio particularmente de:

- La diferencia entre indivisibles e infinitesimales a la hora de calcular una cuadratura, entre otras cosas, para ilustrar el problema de heterogeneidad.

- Las condiciones de convergencia de una serie, que posiblemente ellos reconocieron, pero no enunciaron de manera explícita.

- La búsqueda de resultados utilizando mecanismos geométricos, algebraicos o aritméticos, que usualmente sólo se obtienen mediante el uso del cálculo integral; caso particular de esto se presenta en el trabajo de Cavalieri, en el que a partir de hacer comparaciones entre indivisibles geométricos establece quizá por primera vez el resultado .

$$
\int_{0}^{a} x^{n} d x=\frac{a^{n+1}}{n+1}
$$

- La búsqueda de caminos alternativos para calcular una integral definida que no se base en el método usual de Riemann, es decir, en utilizar rectángulos infinitesimales de igual base; uno de estos caminos es el propuesto por Fermat al emplear rectángulos de bases en progresión geométrica.

Cabe resaltar que en relación con la representación en series de potencias de una función aparecen dos problemas (Ferraro y Panza, 2003), el primero que refiere a encontrar el desarrollo en series de potencias de una función dada y el segundo que hace referencia a determinar cuál función corresponde a un desarrollo en series de potencias dado; es claro que en la época abordada por nosotros el primero de estos problemas era el de interés (en los métodos de Fermat se evidencia esto), lo cual contrasta con lo que es usual en el estudio relacionado con series, puesto que el segundo es el de interés.

Por último, sugerimos para posteriores trabajos de investigación dar continuidad a este estudio a partir de abordar problemas, diferentes al de cuadraturas, que permitieron que las series influyeran en la evolución de los conceptos ya referenciados, o de abordar contextos y épocas en las que el estatus de la serie era considerado como objeto matemático, de tal forma que se pueda seguir el rastro de dicha influencia. Consideramos pertinente, además, la elaboración de propuestas didácticas concretas que giren en torno a rescatar los tratamientos y las temáticas que anteriormente se mencionaron. 


\section{Referencias bibliográficas}

Barrios, J. (1995). La geometría de los indivisibles: Buenaventura Cavalieri. Pagina académica de José Barrios García. Extraído en octubre de 2006 desde: http://webpages.ull.es/users/ jbarrios/page/publicaciones.htm

Cantoral, R. (1990). El pensamiento físico en los siglos XVIII y XIX y su relevancia para una didáctica del análisis. Extraído en agosto de 2006 desde: http:// cimate.uagro.mx/cantoral/

Cantoral, R. (2003). La aproximación socioepistemológica a la investigación en matemática educativa: una mirada emergente. Extraído desde: http:// cimate.uagro.mx/cantoral/

Cantoral, R. y Farfán, R. (2004). Desarrollo conceptual del cálculo. México: Thomson.

Cantoral, R., Molina, J. y Sànchez, J. (2003). Socioepistemológia de la predicción. Extraído desde: http://cimate.uagro. $\mathrm{mx} /$ cantoral/

Castañeda, A. (2004). Un acercamiento a la construcción social del conocimiento: estudio de la evolución didáctica del punto de inflexión. Tesis de Doctorado no publicada. México: Cinvestav

Cohen, L., y Manion, L. (1990). Métodos de investigación educativa. Madrid: Editorial La Muralla.

Ferraro, G., y Panza, M. (2003). Developing into series and returning from series. A note on the foundation of eighteenth-century analysis. Historia Mathematica, 30, 17-46.

Martínez, G. (2003). Caracterización de la convención matemática como un mecanismo de construcción de conocimiento. El caso de su funcionamiento en los exponentes. Tesis de Doctorado no publicada. México: Cinvestav.

Sfard, A. (1991). On the dual nature of mathematical conceptions: reflections on processes and objects as different sides in the same coin. Educational Studies in Mathematics, 22, 1-36.

\section{Bibliografía complementaria}

Boyer, C. B. (1959). A history of the calculus. Nueva York: Dover Publications.

Boyer, C. B. (1987). Historia de la matemática. Madrid: Alianza Editorial.

Brassinne, E. (1989). Oeuvres mathématiques de P. Fermat et de l'arithmétique de Diophante. Toulouse: Éditions Jacques Gabay.

Bressoud, D. (2002). Was calculus invented in India. The College Mathematics Journal, 33, 2-13. Extraído desde: http://links.jstor.org/sici?sici=07468342\%28200201\%2933\%3A1\%3C2\%3 AWCIII\%3E2.0.CO\%3B2-5

Cantoral, R. y Farfán, R. (1990). Elementos metodológicos para la reconstrucción de una didáctica del análisis en el nivel superior. Extraído desde: http://cimate.uagro.mx/cantoral/

Castro, I. y Pérez, H. (2004). Didáctica arquimediana. Memorias del XV Encuentro de Geometría y sus Aplicaciones, Bogotá, junio.

Edwards, C. H. (1982). The historical development of the calculus. Nueva York: Springer-Verlag.

Ferrari, M. (2001). Una visión socioepistemológica. Estudio de la función logaritmo. Tesis de Maestría no publicada. México: Centro de Investigación y de Estudios Avanzados del Instituto Politécnico Nacional.

González, P. M. (2006). Las técnicas del cálculo: Fermat, Wallis y Roberval. Actas Seminario Oratova, 2. Extraído en noviembre de 2006 desde: http:// www.gobiernodecanarias.org/educacion/fundoro/pub_actas2.htm

Kline, M. (1992). El pensamiento Matemático de la antigüedad a nuestros días (vol. 1 y 2). Madrid: Editorial Alianza. 